\title{
Cardiorespiratory Response to Moderate Hypercapnia in Female College Students Expressing Behaviorally Inhibited Temperament
}

\author{
Paul F. Martino 1,2, Daniel P. Miller ${ }^{3}$, Justin R. Miller' ${ }^{2}$, Michael T. Allen', \\ Denise R. Cook-Snyder ${ }^{1,2}$, Justin D. Handy ${ }^{5}$ and Richard J. Servatius ${ }^{6,7 *}$ \\ ${ }^{1}$ Biology Department, Carthage College, Kenosha, WI, United States, ${ }^{2}$ Department of Physiology, Medical College \\ of Wisconsin, Milwaukee, WI, United States, ${ }^{3}$ Neuroscience Department, Carthage College, Kenosha, WI, United States, \\ ${ }^{4}$ School of Psychological Sciences, College of Education and Behavioral Sciences, University of Northern Colorado, Greeley, \\ CO, United States, ${ }^{5}$ Naval Submarine Medical Research Laboratory, Groton, CT, United States, ${ }^{6}$ United States Department \\ of Veterans Affairs, Syracuse VA Medical Center, Syracuse, NY, United States, ${ }^{7}$ Department of Psychiatry, State University \\ of New York Upstate Medical University, Syracuse, NY, United States
}

OPEN ACCESS

Edited by:

Julian F. Thayer,

The Ohio State University,

United States

Reviewed by:

Joao Paulo Jacob Sabino,

Federal University of Piauí, Brazil Luciane H. Gargaglioni,

São Paulo State University, Brazil

${ }^{*}$ Correspondence:

Richard J. Servatius richard.servatius@va.gov

Specialty section:

This article was submitted to Autonomic Neuroscience,

a section of the journal

Frontiers in Neuroscience

Received: 29 July 2020 Accepted: 26 October 2020 Published: 13 November 2020

Citation:

Martino PF, Miller DP, Miller JR, Allen MT, Cook-Snyder DR, Handy JD and Servatius RJ (2020)

Cardiorespiratory Response to Moderate Hypercapnia in Female College Students Expressing

Behaviorally Inhibited Temperament.

Front. Neurosci. 14:588813.

doi: $10.3389 /$ fnins.2020.588813
Behaviorally inhibited $(\mathrm{BI})$ temperament is marked by heightened behavioral sensitivity to environmental threats. The degree to which threat sensitivity is reflected in cardiorespiratory responses has been relatively unexplored. Female college students were exposed to modest hypercapnia $\left(7.0 \% \mathrm{CO}_{2}\right)$ or ambient air (AA) while engaging in a computerized task with cued reinforcement features. All physiological variables except for blood pressure were processed in 4 min epochs corresponding to preexposure, exposure, and post-exposure. Primary respiratory measures were respiratory frequency $\left(\mathrm{f}_{\mathrm{b}}\right)$, tidal volume $\left(\mathrm{V}_{\mathrm{T}}\right)$, and minute ventilation $\left(\mathrm{V}_{\mathrm{E}}\right)$. Electrocardiograms (ECGs) were processed using ARTiiFACT software with resultant heart rate variability (HRV) measures in the frequency domain and time domain. Consistent with the literature, modest hypercapnia increased $V_{T}, F_{b}$, and $V_{E}$. No differences in respiratory parameters were detected between $\mathrm{BI}$ and non-behaviorally inhibited individuals (NI). For HRV in the time domain, RMSSD and NN50 values increased during $\mathrm{CO}_{2}$ inhalation which then returned to pre-exposure levels after $\mathrm{CO}_{2}$ cessation. Hypercapnia increased high frequency (HF) power which then recovered. Bl exhibited reduced low frequency (LF) power during the pre-exposure period. For NI, LF power reduced over the subsequent phases ameliorating differences between $\mathrm{Bl}$ and $\mathrm{NI}$. Hypercapnia improved the task performance of $\mathrm{Bl}$. This is the largest study of female reactivity to hypercapnia and associated HRV to date. In general, hypercapnia increased time domain HRV and HF power, suggesting a strong vagal influence. Those expressing Bl exhibited similar respiratory and HRV reactivity to $\mathrm{NI}$ despite inherently reduced LF power. Although $7 \% \mathrm{CO}_{2}$ represents a mild challenge to the respiratory and cardiovascular systems, it is nonetheless sufficient to explore inherent difference in stress reactivity in those vulnerable to develop anxiety disorders.

Keywords: diatheses, temperament, stress, anxiety, heart rate variability, SDNN, vagal activity 


\section{INTRODUCTION}

Anxiety disorders and posttraumatic stress disorder (PTSD) are best understood as a dynamic interaction of aversive experiences in those with inherent vulnerabilities to develop anxious states (Barlow, 2000; Chasiropoulou et al., 2019). A learning diathesis model accentuates individual differences in associative learning as a final common path to pervasive avoidance (Servatius et al., 2008; Beck et al., 2010; Allen et al., 2019), a core feature of anxiety disorders (Mellick et al., 2019), and PTSD (O’Donnell et al., 2007). The process by which avoidance develops may be through the individual differences in perceived aversiveness, the associativity of aversive experiences/ideations or both.

\section{Behaviorally Inhibited Temperament as a Vulnerability}

Behaviorally inhibited (BI) temperament is one such vulnerability. BI is defined as a personality disposition marked by extreme withdrawal in the face of novel non-social or social challenges (Gladstone and Parker, 2005), with numerous studies linking BI to anxiety disorders (Biederman et al., 2001; Gladstone et al., 2005) and PTSD (Myers et al., 2012a,b; Servatius et al., 2017; Handy et al., 2018). Those individuals with BI temperament display associative learning biases, evident as facilitated acquisition of the classically conditioned eyeblink responses in adolescents (Holloway et al., 2012; Caulfield et al., 2015), adults (Allen et al., 2014, 2016), active duty military (Handy et al., 2018), and veterans (Myers et al., 2012a). The psychophysiological basis of associative learning biases in $\mathrm{BI}$ individuals is unclear. One line of reasoning focuses on attentional processes as a predisposing factor in learning (Mcauley et al., 2009). Those with BI temperament have difficulty disengaging attention from novel stimuli or stimuli associated with threat or distress (Pérez-Edgar et al., 2010; Ricart et al., 2011; Fox and Pine, 2012; Holloway et al., 2012; White et al., 2017).

\section{HRV as a Physiological Source of Vulnerability}

A common substrate for hypervigilance and associative learning biases is the autonomic nervous system (Friedman, 2007), composed of the sympathetic and parasympathetic branches. Heart rate variability (HRV) is a means of assessing contributions of these two branches through decomposition of successive beat-to-beat variations in heart rate (peak $\mathrm{R}$ wave to $\mathrm{R}$ wave within the QRS complexes of electrocardiograms) (Berntson et al., 1997). HRV is partitioned in time and frequency domains. For the frequency domain, accepted bands are low frequency (LF; 0.04-0.15 Hz) and high frequency (HF; 0.15-0.4 Hz), the latter corresponding to the vagal influences on respiration (i.e., respiratory sinus arrhythmia). In the time domain, three measures are commonly evaluated: the standard deviation of normal to normal (NN) intervals (SDNN), root mean square of successive differences (RMSSD) between normal heartbeats, and the number of pairs of successive NNs that differ by more than $50 \mathrm{~ms}$ (NN50). Decreased HRV is evident as reduced LF power and increased HF power in the frequency domain and greater
SDNN, RMSSD, and NN50 in the time domain. In general, better sustained attention or vigilance (Luque-Casado et al., 2016) and facilitated associative learning (Tapp et al., 1997) is associated with decreased HRV.

Further, temperaments with inhibitory features have inherently low HRV. For example, individuals expressing harm avoidance display reduced low frequency power (Puttonen et al., 2008; Huang et al., 2013). Moreover, distressed (Type D) personality, a combination of negative affect and social inhibition linked to cardiovascular disease, has been reported to have low HRV (Martin et al., 2010; Bibbey et al., 2015). Both harm avoidance (Allen et al., 2017) and Type D personality (Servatius et al., 2017; Allen et al., 2018; Handy et al., 2018) are highly correlated with BI temperament. Thus, it is reasonable to expect BI to have inherently reduced HRV or alternatively greater reductions of HRV from exposure to challenges.

\section{Experimental Induced Hypercapnia as a Challenge to HRV}

Experimentally induced hypercapnia has a long history as a potent psychophysiological challenge. Panic-like responses are elicited by high concentrations of $\mathrm{CO}_{2}$ delivered in bolus (e.g., $35 \% \mathrm{CO}_{2}$ ) (Van Den Hout et al., 1987; Perna et al., 2003) or lower concentrations (e.g., $5 \% \mathrm{CO}_{2}$ ) delivered over extended exposure (e.g., $20 \mathrm{~min}$ ) (Gorman et al., 1988; Bystritsky et al., 2000; Valenca et al., 2002). Such challenge parameters are quite demanding and may narrow the range in which to observe differences in those vulnerable to anxiety disorders.

Lower levels of $\mathrm{CO}_{2}$ delivered over shorter durations are more tolerable and produce gradations in demands placed on the system to adjust to hypercapnia from simple increases in tidal volume $\left(\mathrm{V}_{\mathrm{t}}\right)$ (Brown et al., 2014) to increased $\mathrm{V}_{\mathrm{t}}$ accompanied by elevated HR (Tzeng et al., 2007). Even at mild levels of hypercapnia, the attendant acidosis drives deeper breathing through central chemoreceptors (Brown and Howden, 2008a). In terms of HRV, mild hypercapnia increases HF power (Brown et al., 2007, 2014; Tzeng et al., 2007). Although HF HRV is closely linked to vagal activity and respiratory sinus arrhythmia (RSA) in general, increased HF power secondary to hypercapnia may be dissociated from RSA (Brown et al., 2014). To date, time domain measures of HRV have been unaffected by mild hypercapnia (Brown et al., 2007). Thus, mild hypercapnia is potent enough to affect both respiratory parameters and HRV, particularly in the frequency domain.

\section{The Present Study}

The present study examined respiratory and cardiovascular reactivity to mild hypercapnia as a function of BI temperament. Hypercapnia was expected to increase HF power. The Adult Measure of Behavioral Inhibition (AMBI) (Gladstone and Parker, 2005) was used to classify individuals as either BI or noninhibited (NI). Those expressing BI were expected to exhibit reduced HRV. We further expected BI individuals to express more reactivity to mild hypercapnia. After a baseline period, participants engaged in a computerized task that requires attention; those expressing BI tend to perform better than NI 
on the task. Onset and terminations of the computer task were contemporaneous with introduction of hypercapnia and its return to ambient air (AA). The computerized task was used as a behavioral indicant of stress reactivity inasmuch as attention to hypercapnic challenge could be manifest as poorer performance on the task through divided attention. While male BI participants tend to perform better than male NI participants, BI females tend to be similar to NI females (Sheynin et al., 2014). Further, females are largely underrepresented in the study of mild hypercapnia and HRV (Brown et al., 2007; Tzeng et al., 2007). Therefore, we restricted this initial study to female participants.

\section{MATERIALS AND METHODS}

\section{Participants and Recruitment}

Undergraduate volunteers were recruited at Carthage College, Kenosha, WI, United States. Ninety-nine females with an age range of 18-22 years voluntarily completed the study. Participants with a self-reported history of psychiatric illnesses, neurological disorders, cardiac disorders, or respiratory illnesses were excluded from the study. Participants were asked to refrain from drinking alcohol or chewing, smoking, or vaping any tobacco products for $24 \mathrm{~h}$ prior to the start of the study. Additionally, participants were asked to refrain from eating, exercising, or drinking caffeinated beverages for $2 \mathrm{~h}$ prior to the study. All eligible participants completed an informed consent agreement upon arrival for their scheduled study appointments and were given the opportunity to ask questions before initiating study procedures. Participants were not compensated for their participation, but received partial credit toward academic research requirements. The study was approved by the Institutional Review Board of Carthage College (IRB Approval \#: 1133068-4) in compliance with all applicable Federal regulations governing protection of human subjects.

\section{Experimental Design}

Participants were randomly assigned to receive either room ambient air (AA groups) through the breathing apparatus or $7 \% \mathrm{CO}_{2}$ (7\% $\mathrm{CO}_{2}$ groups). There were three phases: Baseline, Exposure, and Recovery. The Exposure and Recovery phases were $4 \mathrm{~min}$ each. The Baseline period was $15 \mathrm{~min}$, but only the last $4 \mathrm{~min}$ were evaluated to equate with the other two phases for all physiological parameters. The exposure groups were characterized as NI or BI, as elaborated below. Thus, a $2 \times 2$ design was constructed: AA-NI $(N=29)$, AA-BI $(N=16), 7 \%$ $\mathrm{CO}_{2}$ - $\mathrm{NI}(\mathrm{N}=32)$, and $7 \% \mathrm{CO}_{2}-\mathrm{BI}(N=21)$. During informed consent, participants were advised that exposure to hypercapnia was a possibility, but subjects were otherwise blind to assignment. With respect to behavioral inhibition, investigators were blind to the condition.

\section{Scales}

All participants completed the Adult Measure of Behavioral Inhibition (AMBI) which consists of 16 items probing aspects of BI temperament (Gladstone and Parker, 2005; Gladstone et al., 2005). Items assess the degree to which participants exhibit inhibited or avoidant behaviors in new or unfamiliar social and non-social situations on a 3-point Likert-type scale. Possible scores range from 0 to 32 with higher scores indicating higher levels of BI. Consistent with previously established methodology (Allen et al., 2014; Servatius et al., 2017; Handy et al., 2018), individuals were classified as NI or BI based on a cut score of 15.5.

\section{Computerized Task}

Participants were presented with a computerized game to measure attention and performance during modest hypercapnia. The task was adapted from a spaceship game in which a participant defends a base from incoming spaceships which drop bombs, which has been more completely described elsewhere (Sheynin et al., 2015). The software was programmed in SuperCard version 3.7.1 (Solutions Etcetera, Pollock Pines, CA, United States) and presented on a Macintosh iMac computer. The keyboard was masked except for three keys labeled FIRE, LEFT, and RIGHT, which the subject could use to enter responses. Briefly, a participant began with 325 points which could increase or decrease based on performance. The game began with exposure and lasted $8 \mathrm{~min}$.

\section{Gas Preparation, Delivery, Measurement}

The custom system for gas preparation and delivery employed in the present study was previously described (Miller et al., 2018). Ambient air (AA) was mixed with medical grade $\mathrm{CO}_{2}$ using a 13.5 L respirometer/spirometer (P-1300, Warren E. Collins, Inc., Braintree, MA, United States) to achieve a concentration of 7\% $\mathrm{CO}_{2}$. This was verified through capnography using an $\mathrm{O}_{2} \mathrm{CAP} \mathrm{O} 2$ and $\mathrm{CO}_{2}$ analyzer (Oxigraf, 07-7021). Once the gases were mixed, the mixture was transferred from the respirometer/spirometer to $300 \mathrm{~g}$ weather balloons, which could store up to $1600 \mathrm{~L}$ of gases for at least $1 \mathrm{~h}$ without any change in the concentrations of gases.

\section{Experimental Procedures}

Data collection occurred during one of two 1-h time slots at 1400 and 1500 h 5 days a week (Monday-Friday). All participants were seated in the upright position in front of a computer desk which displayed a computerized task described above. Upon signing informed consent, blood pressure measurements were taken while seated upright with both arms resting on the deck in front of them, using a Leader fully automated blood pressure monitor (BP-3AG1-1PLDR, Cardinal Health). Subjects were outfitted with a face mask system that covered both the mouth and the nose and was connected to a two-way nonrebreathing valve and pneumotach (Model 3813, Hans Rudolph, Shawnee, KS, United States). Participants were also instrumented for ECG collection. Baseline acclimation to the apparatus was 15 min. During the acclimation period participants completed the demographic data and scales. At the end of the baseline period, the computerized task began. The task period was 8-min. During the first 4-min task period participants were exposed to AA or $7 \% \mathrm{CO}_{2}$. During the last 4-min task period all participants were exposed to AA. Upon the task completion a second blood pressure measure was obtained. 


\section{Respiration and ECG Collection and Processing}

Airflow as minute ventilation $\left(\mathrm{V}_{\mathrm{E}} ; \mathrm{L} / \mathrm{min}\right)$, breathing frequency (fb; breaths/min), and tidal volume $\left(\mathrm{V}_{\mathrm{T}} ; \mathrm{L}\right)$ were all measured with an airflow transducer and digital data acquisition module (Bio Pac MP36 and MP150, Goleta, CA, United States).

Three-lead electrocardiogram (ECG) was collected at $100 \mathrm{~Hz}$ with BIOPAC models (ECG 100C and MEC 110C) and digital data acquisition module (MP150). ECGs were extracted for the 4 min prior to start of task, 4 min of exposure $\left(7 \% \mathrm{CO}_{2}\right.$ or AA), and $4 \mathrm{~min}$ of recovery. Subjects without complete records for the all three 4-min epochs were excluded from analysis. ECG was processed with ARTiiFACT software (Kaufmann et al., 2011). Interbeat intervals were computed from identified and visualized $\mathrm{R}$ waves. Artifacts were identified through Berntson detection and cubic spline correction. HRV parameters were computed with standard values of $4 \mathrm{~Hz}$ interpolation rate and $50 \%$ window overlap with standard frequency bands $0.04,0.15$, and 0.4 parameters. The very low frequency band $(0.04-0.15 \mathrm{~Hz})$ was otherwise ignored. Consistent with the literature, the normalized value of power (nu) as well as the natural $\log (\mathrm{ln})$ of raw power of LF and HF were analyzed to interpret power changes attributable to a particular band (Quintana and Heathers, 2014; Quintana et al., 2016). nuLF and nuHF are complementary, thus analysis of one is sufficient to understand power has changed with experimental conditions, but still not specific to either band. Analysis of the raw power is necessary to understand the nature of changes in nuLF/nuHF (Heathers, 2014).

\section{Analytic Approach}

All statistical analyses were performed in IBM SPSS Statistics for Windows, version 26 (IBM Corp., Armonk, NY, United States) and R (R Core Team, 2020, Vienna, Austria). Respiratory measures and HRV measures were subjected to separate 2 (Exposure; AA vs. $\left.\mathrm{CO}_{2}\right) \times 2$ (Temperament; NI vs. $\left.\mathrm{BI}\right) \times 3$ [Phase; Baseline (B), Exposure (E), and Recovery (R)] mixed analyses of variance (ANOVAs) with $p<0.05$. Blood pressure measured prior to baseline recording and after recovery was analyzed with Exposure $\times$ Temperament $\times$ Time (pre vs. post) mixed ANOVA. Behavioral performance was analyzed with Exposure $\times$ Temperament multivariate ANOVA.

\section{RESULTS}

\section{Respiratory Parameters}

Breathing frequency increased in all participants during the Exposure phase, which corresponded with the start of the computerized task. Breathing frequency increased from 14 breathes/min during baseline to $16-17$ breathes/min during the Exposure and Recovery phases (See Table 1). The main effect of Phase was significant, $F(2,178)=87.3 ; \eta_{\mathrm{p}}{ }^{2}=0.58, p<0.001$. As expected, hypercapnia specifically increased $V_{T}, V_{E}$, and $\mathrm{V}_{\mathrm{I}}$. Exposure $\times$ Phase interactions were appear for both $\mathrm{V}_{\mathrm{T}}$, $F(2,178)=23.6 ; \eta_{\mathrm{p}}{ }^{2}=0.21$, and $\mathrm{V}_{\mathrm{E}}, F(2,178)=35.8 ; \eta_{\mathrm{p}}{ }^{2}=0.29$, all $p$ 's $<0.002$ (See Table 1 ). In addition, the time of inspiration
$\left(\mathrm{T}_{\mathrm{I}}\right)$ and expiration $\left(\mathrm{T}_{\mathrm{E}}\right)$ were analyzed. Hypercapnia induced shorter $\mathrm{V}_{\mathrm{I}}$ without altering $\mathrm{V}_{\mathrm{E}}$. For the $\mathrm{V}_{\mathrm{i}}$, an Exposure $\times$ Phase interaction, $F(2,178)=6.6, \eta_{\mathrm{p}}{ }^{2}=0.07$. Although hypercapnia induced quicker inhalation with greater depth, there were no main effects or interactions with BI.

\section{HR and HRV}

Analysis of HR only yielded a significant main effect of Phase, $F(2,178)=11.5, \eta_{\mathrm{p}}{ }^{2}=0.115$. HR generally decreased from Baseline to the Exposure phase. Accordingly, average RR intervals generally increased from the Baseline to the Exposure phase (See Table 1). The exposure phase was coincident with the start of the computerized task, thus general phases changes in HR and RR intervals may reflect attention to the task.

For the time domain, Exposure $\times$ Phase interactions were evident for $\operatorname{SDNN}, F(2,178)=4.1, p=0.02, \eta_{\mathrm{p}}{ }^{2}=0.04$, RMSSD, $F(2,178)=6.5, p=0.002, \eta_{\mathrm{p}}^{2}=0.07$, and NN50, $F(2,178)=9.7, p<0.001, \eta_{\mathrm{p}}{ }^{2}=0.10$ (see Figure 1). For each,

TABLE 1 | Respiratory and cardiovascular parameters over experiment epochs within ambient air (AA) and $7 \% \mathrm{CO}_{2}$ conditions in $\mathrm{BI}$ and $\mathrm{NI}$ participants.

\begin{tabular}{|c|c|c|c|}
\hline & Baseline & Exposure & Recovery \\
\hline \multicolumn{4}{|l|}{ AA-NI } \\
\hline $\mathrm{HR}$ & $77.8 \pm 2.1$ & $75.5 \pm 2.1$ & $77.9 \pm 2.1$ \\
\hline Mean RR & $786.2 \pm 20.6$ & $809.9 \pm 21.2$ & $784.0 \pm 20.6$ \\
\hline Breathing frequency (b/min) & $13.8 \pm 0.7$ & $16.0 \pm 0.7^{\star}$ & $16.6 \pm 0.7$ \\
\hline $\mathrm{V}_{\mathrm{T}}(\mathrm{L})$ & $0.9 \pm 0.04$ & $0.8 \pm 0.04$ & $0.7 \pm 0.03$ \\
\hline$V_{E}(\llcorner/ \min )$ & $11.3 \pm 0.5$ & $11.7 \pm 0.5$ & $11.5 \pm 0.4$ \\
\hline $\mathrm{T}_{1}(\mathrm{~s})$ & $2.6 \pm 0.1$ & $2.5 \pm 0.1$ & $2.4 \pm 0.1$ \\
\hline $\mathrm{T}_{\mathrm{E}}(\mathrm{s})$ & $1.1 \pm 0.1$ & $1.0 \pm 0.1$ & $0.9 \pm 0.1$ \\
\hline \multicolumn{4}{|l|}{ AA-BI } \\
\hline $\mathrm{HR}$ & $78.4 \pm 2.7$ & $75.4 \pm 2.7$ & $77.8 \pm 2.7$ \\
\hline Mean RR & $777.6 \pm 25.9$ & $808.3 \pm 26.7$ & $782.6 \pm 25.9$ \\
\hline Breathing frequency $(\mathrm{b} / \mathrm{min})$ & $14.2 \pm 0.9$ & $17.3 \pm 0.8^{\star}$ & $17.2 \pm 0.8$ \\
\hline $\mathrm{V}_{\mathrm{T}}(\mathrm{L})$ & $0.8 \pm 0.06$ & $0.7 \pm 0.05$ & $0.6 \pm 0.04$ \\
\hline$V_{E}(\llcorner/ \min )$ & $10.6 \pm 0.6$ & $10.9 \pm 0.6$ & $10.7 \pm 0.5$ \\
\hline $\mathrm{T}_{1}(\mathrm{~s})$ & $2.7 \pm 0.1$ & $1.8 \pm 0.1^{*}$ & $2.1 \pm 0.1^{*}$ \\
\hline$T_{E}(s)$ & $1.3 \pm 0.1$ & $0.9 \pm 0.1$ & $0.9 \pm 0.1$ \\
\hline \multicolumn{4}{|l|}{$7 \% \mathrm{CO}_{2}-\mathrm{NI}$} \\
\hline $\mathrm{HR}$ & $81.0 \pm 2.0$ & $79.6 \pm 2.0$ & $81.9 \pm 2.0$ \\
\hline Mean RR & $750.6 \pm 19.5$ & $765.6 \pm 20.1$ & $745.6 \pm 19.5$ \\
\hline Breathing frequency $(\mathrm{b} / \mathrm{min})$ & $13.9 \pm 0.7$ & $16.9 \pm 0.6^{\star}$ & $17.3 \pm 0.6$ \\
\hline $\mathrm{V}_{\mathrm{T}}(\mathrm{L})$ & $0.9 \pm 0.05$ & $1.0 \pm 0.04^{*}$ & $0.8 \pm 0.03$ \\
\hline$V_{E}(\llcorner/ \min )$ & $11.2 \pm 0.4$ & $15.4 \pm 0.5^{\star}$ & $13.3 \pm 0.3$ \\
\hline $\mathrm{T}_{1}(\mathrm{~s})$ & $2.8 \pm 0.2$ & $2.3 \pm 0.1$ & $2.4 \pm 0.1$ \\
\hline$T_{E}(s)$ & $1.0 \pm 0.2$ & $0.8 \pm 0.1$ & $1.0 \pm 0.1$ \\
\hline \multicolumn{4}{|l|}{$7 \% \mathrm{CO}_{2}-\mathrm{BI}$} \\
\hline $\mathrm{HR}$ & $80.8 \pm 2.5$ & $80.5 \pm 2.5$ & $81.3 \pm 2.5$ \\
\hline Mean RR & $764.0 \pm 24.5$ & $765.1 \pm 25.3$ & $756.4 \pm 24.5$ \\
\hline Breathing frequency (b/min) & $14.1 \pm 0.8$ & $16.5 \pm 0.8^{\star}$ & $17.5 \pm 0.8$ \\
\hline $\mathrm{V}_{\mathrm{T}}(\mathrm{L})$ & $0.9 \pm 0.06$ & $1.0 \pm 0.05^{*}$ & $0.8 \pm 0.04$ \\
\hline$V_{E}(L / \min )$ & $11.1 \pm=0.5$ & $15.9 \pm 0.6^{\star}$ & $13.5 \pm 0.4$ \\
\hline $\mathrm{T}_{1}(\mathrm{~s})$ & $2.5 \pm 0.2$ & $1.8 \pm 0.1^{\star}$ & $2.0 \pm 0.1^{*}$ \\
\hline$T_{E}(s)$ & $1.0 \pm 0.2$ & $0.9 \pm 0.1$ & $0.8 \pm 0.1$ \\
\hline
\end{tabular}

* indicates significant change from Baseline, $p<0.05$. 
HRV increased during hypercapnia whereas increases were not evident in those given AA. For SSDN, the AA group reduced vagal tone across the experimental phases. There were no main effects or interactions with BI.

For the frequency domain, normalized frequencies (nuLF or nuHF) yielded significant interactions of Exposure $\times$ Phase, $F(2,178)=3.5, p=0.03 ; \eta_{\mathrm{p}}{ }^{2}=0.04$ (See Figure 2, left panel) and BI $\times$ Phase, $F(2,178)=3.8, p=0.02 ; \eta_{\mathrm{p}}{ }^{2}=0.04$, (See Figure 2, right panel). Interpretation of normalized power requires analysis of the individual $\operatorname{lnLF}$ and $\ln \mathrm{HF}$ power. An analysis of $\ln \mathrm{HF}$ power indicated an Exposure $\times$ Phase interaction, $F(2,178)=6.4$, $p=0.002 ; \eta_{\mathrm{p}}{ }^{2}=0.07$ (See Figure 3, left panel), without significant effects related to $\mathrm{BI}$. This supports the interpretation that differences in normalized power attributable to $\mathrm{CO}_{2}$ exposure reflected an increase in $\mathrm{HF}$ power. Whereas an analysis of $\operatorname{lnLF}$ power showed a BI $\times$ Phase interaction, $F(2,178)=3.4$, $p=0.03 ; \eta_{\mathrm{p}}{ }^{2}=0.034$, without effects of Exposure (See Figure 3, right panel). This supports the interpretation that $\mathrm{BI} \times$ Phase interaction in normalized power was due to decreased $\operatorname{lnLF}$.

\section{Blood Pressure}

Blood pressure, measured before donning the mask and after its removal, did not change as a function of Exposure, BI or time, all $p$ 's $>0.35$. Mean arterial pressure before the experiment was $92.4 \pm 0.9 \mathrm{mmHg}$ and $91.9 \pm 1.4 \mathrm{mmHg}$ after.

\section{Behavior}

There were significant $\mathrm{BI} \times$ Exposure interactions for shots fired, $F(1,81)=4.04, \eta_{\mathrm{p}}{ }^{2}=0.04, p=0.048$, and total points scored, $F(1,81)=4.63, \eta_{\mathrm{p}}{ }^{2}=0.54, p=0.034$. No significant effects were noted for screen movement. For NI individuals, exposure to $\mathrm{CO}_{2}$ tended to inhibit performance, whereas $\mathrm{BI}$ individuals exposed to $\mathrm{CO}_{2}$ performed better (See Figure 4).

\section{DISCUSSION}

The current protocol examined respiratory and cardiovascular adaptations to moderate hypercapnia in female BI and NI undergraduates while performing an engaging computerized task.

\section{Task-Related Adjustments}

The task, a space-based game, was coincidental with the exposure period. Therefore, changes in respiratory and cardiovascular parameters apparent in both $\mathrm{AA}$ and $7 \% \mathrm{CO}_{2}$ groups are interpreted as adaptations to attending to the task and task performance. Such general changes were apparent as a modest bradycardia and increased respiratory rate. It is against this background that effects from hypercapnia are evaluated.

\section{Hypercapnia Induces Modest Respiratory Adjustments}

Overall, hypercapnia induced modest but robust adjustments in respiratory parameters measured. Hypercapnia increased $V_{t}$ and increased $V_{E}$, compared to the participant's baseline and relative to those given $\mathrm{AA}$. Ti also decreased during $7 \% \mathrm{CO}_{2}$ exposure. The pattern of adjustments in respiration parameters are similar to the work of others (Brown et al., 2007; Tzeng et al., 2007), although the magnitude of changes in $\mathrm{V}_{\mathrm{T}}$ appear less which may reflect differences in the composition of the participant pool.

\section{Hypercapnia and HRV}

Recording of ECG concomitant with respiratory parameters afforded the opportunity to assess the impact of hypercapnia on HRV. The recording parameters were non-optimal $(100 \mathrm{~Hz}$ sampling frequency over successive 4-min epochs), thus these results need to be understood under this light. Consistent with others, hypercapnia increased nuHF relative to their baseline and the AA group (Brown et al., 2014). Inasmuch as increased nuHF may be a function of increased HF or lowered LF, the individual frequencies were assessed (Laborde et al., 2017). A corresponding interaction showing increased $\ln \mathrm{HF}$ was apparent, supporting the interpretation that hypercapnia increased HF power. Increased HF power in response to hypercapnia is consistent with the work of others (Brown et al., 2007, 2014). Further, hypercapnia induced increases in HRV in the time domain. An increase in vagal power was apparent with $\operatorname{lnSDNN}$, lnRMSSD, and NN50, with a recovery evident in NN50. The increases were apparent with and without respiration rates included as a covariate in the analyses. The increase in HRV was apparent in the presence of an engaging computerized task that was contemporaneous, albeit not exclusive to hypercapnia. Previous work assessing time domain HRV in moderate hypercapnia have not found differences (Brown et al., 2007, 2014). One potential difference may be the body position of individuals during exposure. In previous studies individuals were supine during exposure; in this study, individuals were seated. Inasmuch as time domain measures of HRV are considerably less in the seated compared to supine position (Young and Leicht, 2011), seated exposure may provide a greater dynamic range to observe increases from hypercapnia.

Hypercapnia did not affect HR. A dissociation between HR and HF HRV in hypercapnia has previously been observed (Brown et al., 2007, 2014) with modest exposure to hypercapnia. Analysis of HRs segregated into inspiratory and expiratory beats found very subtle differences between AA and $5 \% \mathrm{CO}_{2}$ inhalation (Brown et al., 2007). Although we did not assess RSA, our exposure represents a similarly mild challenge. Further, there are dissociations between HR, HF HRV and respiratory sinus arrhythmia (RSA) during hypercapnia at low levels of exposure (Tzeng et al., 2007; Brown et al., 2014), suggesting a dissociation between vagal tone and HF HRV.

One potential mechanism for increased $\mathrm{HF}$ power is acidosis secondary to hypercapnia (Brown and Howden, 2008b). Peripheral chemoreceptors (the carotid bodies and secondarily the aortic bodies) along with central chemoreceptors are sensitive to changes in the blood $\mathrm{pH} / \mathrm{CO}_{2}$ tension. Evidence suggests that pontomedullary nuclei (medial and lateral parabrachial nucleus, retrotrapezoid nucleus/parafacial area, the medullary raphe, and the pre-Bötzinger complex) are sensitive to $\mathrm{pH} / \mathrm{CO}_{2}$ tension and signal the phrenic nerve to increase minute ventilation in order to expel excess $\mathrm{CO}_{2}$ (Guyenet et al., 2013). The $\mathrm{pH} / \mathrm{CO}_{2}$ central 

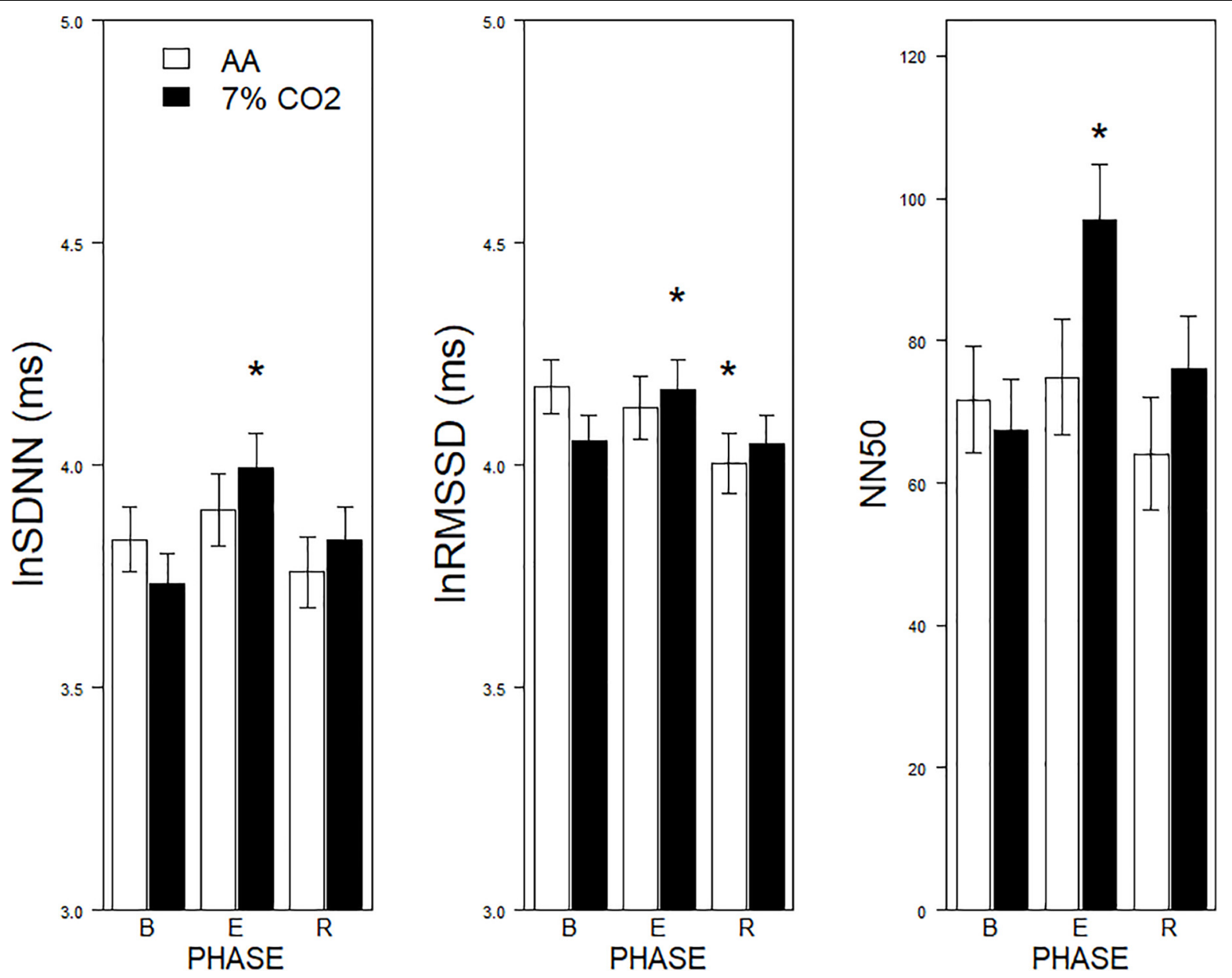

FIGURE 1 | Effects of $7 \% \mathrm{CO}_{2}$ on measures of Time Domain HRV across the three experimental phases. Three measures of the Time Domain in HRV are depicted from left to right panels, SDNN, RMSSSD, and NN50, respectively. The legend for all three panels is contained in the left panel. For all three measures, $*$ indicates a significant change from baseline, $p<0.05$. For NN50, * additionally indicates a difference in recovery, $p<0.05$.
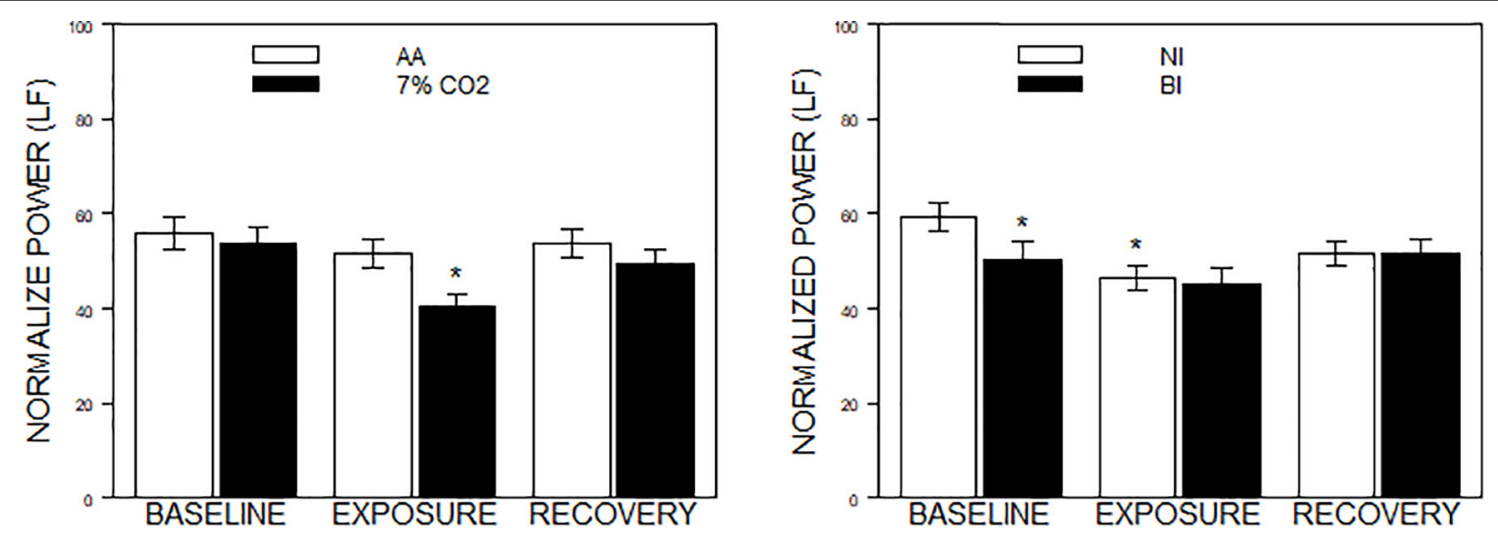

FIGURE 2 | Normalized power in the frequency domain as a function of $7 \% \mathrm{CO}_{2}$ exposure and temperament. The panels depict measures of normalized power from the HF and LF domains across the three experimental phases. Normalized LF was used for the analysis, but note that LF and HF are reciprocal. The legend for each panel is contained within the panel. The left panel depicts the Exposure $\times$ Phase interaction in which normalized LF power decreased during $7 \% \mathrm{CO}_{2}$ exposure which recovered with reintroduction of AA. * indicate $p<0.05$ compared to both baseline values and AA during Exposure. The right panel depicts the $\mathrm{BI} \times$ Epoch interaction. * indicate differences relative to the baseline phase, $p<0.05$. Normalized LF was initially lower in BI than $\mathrm{NI}$. Over participation in the experiment (e.g., computerized task), LF reduced in NI to the levels of BI. 

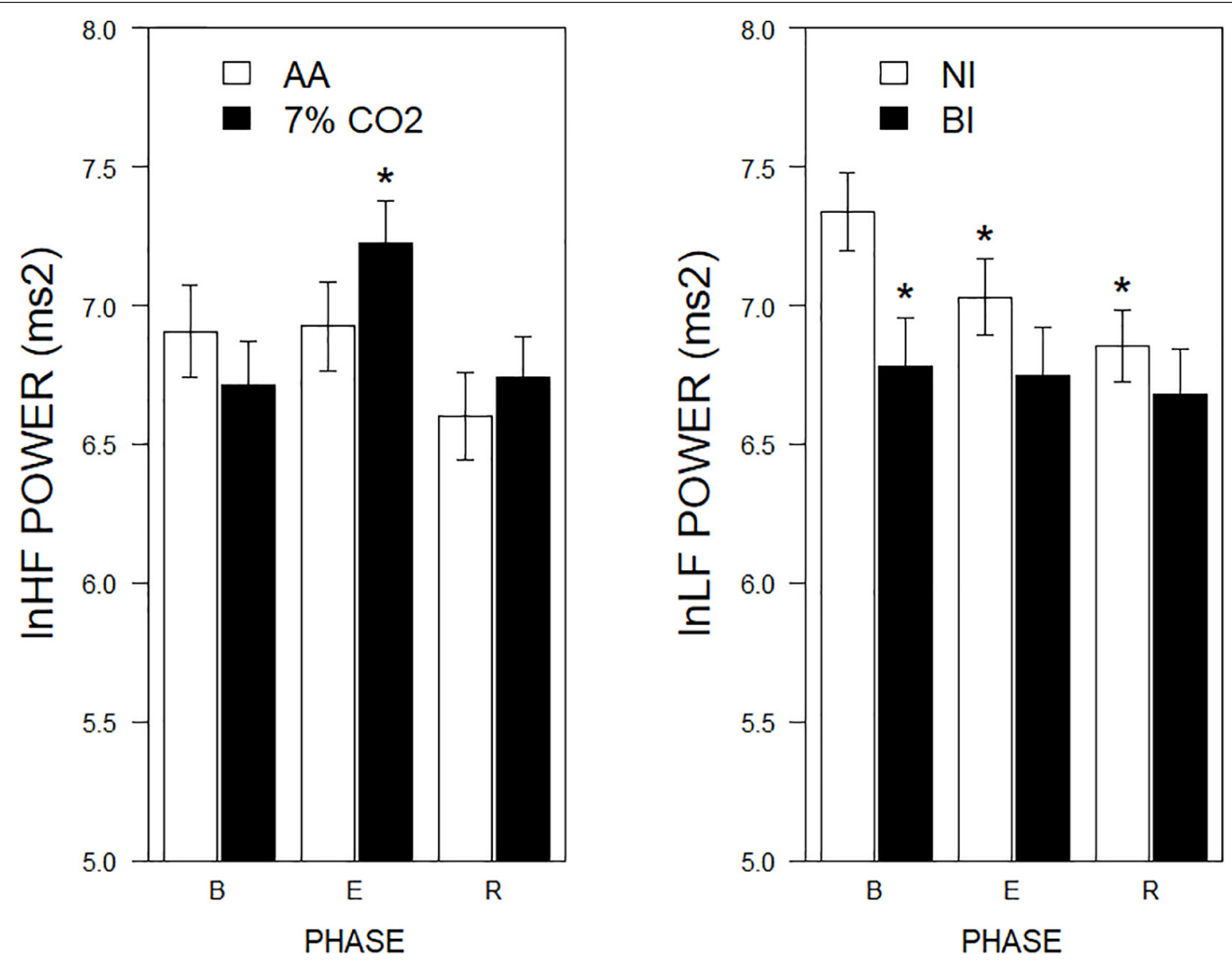

FIGURE 3 | InLF and InHF power across experimental phases as a function of $7 \% \mathrm{CO}_{2}$ exposure and temperament. The right panel depicts InLF power, whereas the left panel depicts InHF power. The legend for each panel is contained within the panel. In the left panel, InHF power increased in $7 \%$ CO 2 relative to baseline and recovery epochs. InHF power did not appreciably change in AA over experimental epochs. This pattern is reminiscent of left panel of Figure 2. In the right panel, InLF power of BI group was initially lower during baseline than NI. LF power of NI reduced over experimental epochs to be similar to that of BI, which did not change from baseline. This pattern is reminiscent of right panel of Figure $2 .{ }^{*}$ indicate a significant change from the respective baseline phase, $p<0.05$.

chemoreceptors drive the increase in activity of the pre-Bötzinger complex (the proposed rhythm generator) eventually stimulating the phrenic nerve, which then drives diaphragm activity, and this increased diaphragm drive ultimately increases breathing. Increased HF power may be secondary to phrenic drive.

Thus, the current protocol and procedures of hypercapnia of $7 \% \mathrm{CO}_{2}$ delivered over a 4 -min period represented a mild physiological challenge.

\section{BI Temperament}

A primary goal of the study was to determine whether BI temperament differed in HRV either as a constituent process and/or as a response to challenge compared to NI individuals. As a constituent process, time domain measures of HRV did not differ between BI and NI individuals. A difference was evident in terms of $\operatorname{lnLF}$ power, with BI individuals displaying lower LF power. The difference between BI and NI diminished over experimental phases. Hypercapnia drove LF power lower in NI, however, hypercapnia did not diminish LF in BI suggesting LF power may have been at nadir in BI individuals. An alternative explanation for the lower baseline LF power is BI individuals are more responsive to the laboratory setting, apparatus or instructions therein (a "white coat" reaction). Arguing against this interpretation, $\mathrm{HR}$ and $\mathrm{BP}$, two responses normally sensitive to laboratory reactivity, were essentially poolable between NI and $\mathrm{BI}$ individuals.

The hypercapnia challenge did not differentially affect the physiological responses of BI participants. The respiratory effects of hypercapnia were no different in BI than NI individuals. Further, there were no exposure-specific differences in HRV in the time or frequency domains. Thus, the inherently low LF power did not manifest in further changes in response to hypercapnia. Note that there was no evidence of panic or paniclike behavioral or physiological responses to hypercapnia in any participant.

A computerized game was presented as a foreground task contemporaneous with $\mathrm{CO}_{2}$ exposure. Participants knew that exposure to $\mathrm{CO}_{2}$ was possible, but did not know when or if they would be exposed. BI individuals could have perceived hypercapnia as stressful, as more of a challenge than NI individuals. If so, one would expect performance on the foreground task to suffer more than NI performance inasmuch as attention would be divided between the hypercapnia and attendant adjustments and the game. Indeed, hypercapnia mildly degraded NI performance. However, performance of BI individuals exposed to hypercapnia was better than 

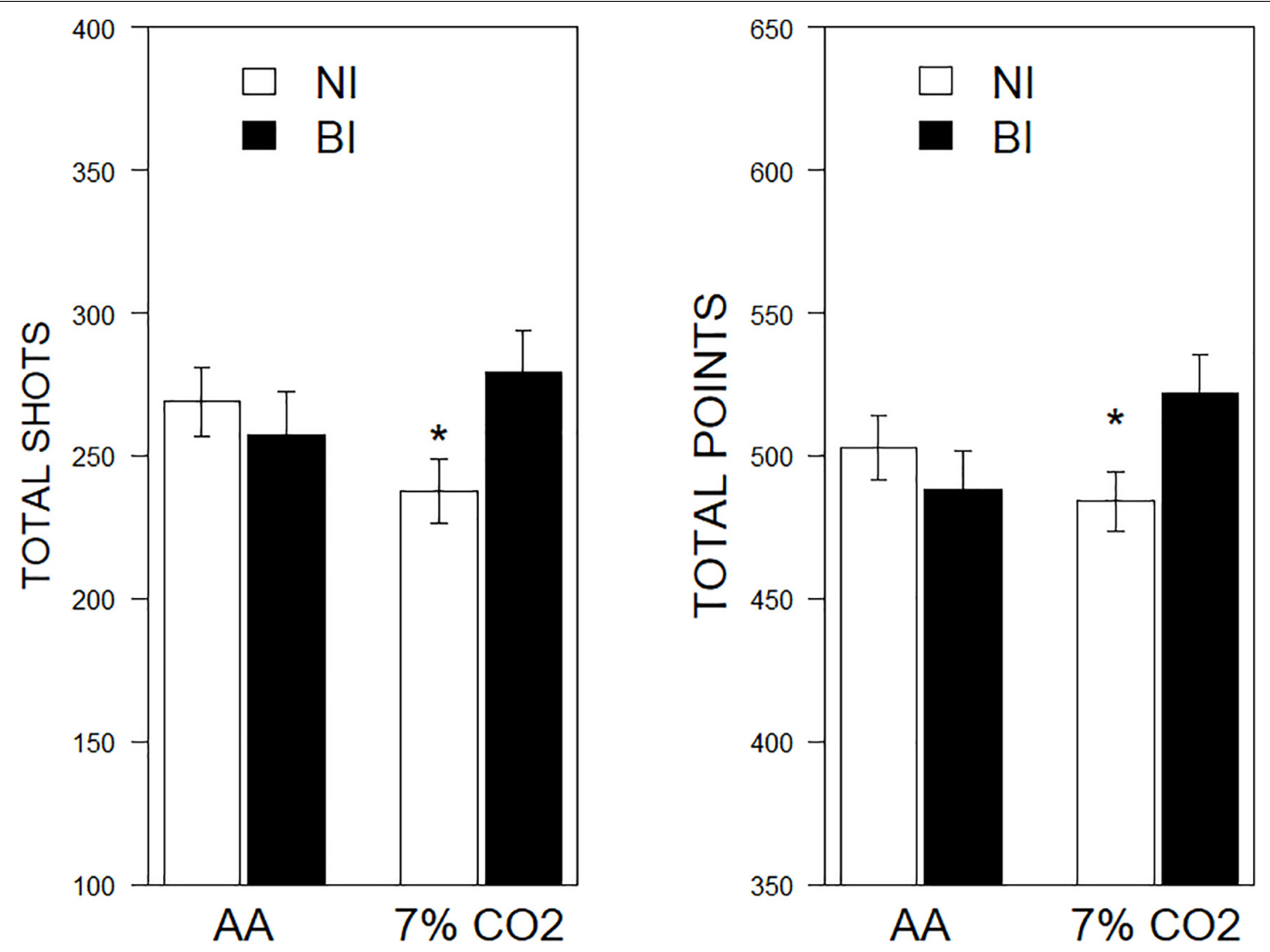

FIGURE 4 | Performance on a computerized game as a function of $7 \% \mathrm{CO}_{2}$ or $\mathrm{AA}$. The legend is contained within the panels. In the left panel, total shots fired are depicted. $\mathrm{NI}$ exposed to $7 \% \mathrm{CO}_{2}$ fired fewer shots than $\mathrm{NI}$ given $\mathrm{AA}$ and $\mathrm{BI}$ exposed to $7 \% \mathrm{CO}_{2}$. The right panel depicts total points scored. Similarly, $\mathrm{NI}$ exposed to $7 \% \mathrm{CO}_{2}$ scored fewer points than $\mathrm{NI}$ given $\mathrm{AA}$ and $\mathrm{BI}$ exposed to $7 \% \mathrm{CO}_{2} .{ }^{*}$ indicate a significant change from the respective $\mathrm{AA}$ group, $p<0.05$.

hypercapnia-exposed NI participants. Performance was not significantly related to any of the HRV parameters examined.

The enhanced performance of BI individuals is reminiscent of learning performances of BI and animal models of BI. In eyeblink conditioning, the unconditional reflex of BI is of similar magnitude to NI, but learning is facilitated (Holloway et al., 2012; Handy et al., 2018). In avoidance motivated by shock, response to the shock per se is similar between models of BI and NI, but avoidance is facilitated (Servatius et al., 2008). While primary physiological responses to challenges may be similar between $\mathrm{BI}$ and NI, the motivational properties to alter behavior largely distinguish BI and NI and by extension vulnerability to develop anxiety disorders. In the face of challenges, BI individuals marshal behavioral and by extension experiential resources. Displacement and focused attention, as exhibited by BI individuals, may be fundamental to anxiety vulnerability. Inherently low LF power may provide the physiological basis for biased performance under modest challenge.

\section{Limitations}

There are several limitations to consider in evaluating the results from the current study. As stated earlier, the parameters for assessing HRV were not optimal. It is recommended that epochs for LF power assessments are $5 \mathrm{~min}$ and sampling rate for
ECG is $200 \mathrm{~Hz}$ (Quintana and Heathers, 2014; Laborde et al., 2017). The finding of inherently low LF power in BI should be replicated with more optimal parameters. The HF component and the time domain measures are well within recommendations. Accordingly, the observed increased HF during hypercapnia is consistent with that in the literature regarding the impact of moderate hypercapnia on HRV (Brown et al., 2007, 2014). It should also be noted that we restricted the study to female participants. Females are generally an understudied population in psychophysiology. In the literature cited concerning hypercapnia and HRV, females comprise about $25 \%$ of those samples with numbers too small to know whether males and females differ in reactivity to hypercapnia. A study with male participants to directly compare and extend the findings is warranted. We also did not record respiration as an independent channel precluding an analysis of RSA. Similarly, blood gases were also not monitored. Both of these measures were challenging within the small undergraduate college environment with the resources available.

\section{Summary}

In summary, mild hypercapnia induces increases in $\mathrm{HF}$ power consistent with the work of others. However, we also observed increased HRV in the time domain in response to 
$7 \% \mathrm{CO}_{2}$ particularly with the measures of RMSSD and NN50. A primary purpose was to evaluate $\mathrm{HRV}$ in $\mathrm{BI}$ as well as in response to the $7 \% \mathrm{CO}_{2}$ challenge. A trait-like reduction in LF power was observed in BI females. Moreover, BI females displayed enhanced reactivity to $7 \% \mathrm{CO}_{2}$ with improved performance on the computerized task. However, the respiratory responses to hypercapnia as well as HRV reactivity were similar between BI and NI. To the degree which inherently lower LF power contributes to vulnerability needs to be elaborated. These data contribute to an understanding of vulnerability to develop anxiety disorders in keeping with a learning diathesis model.

\section{DATA AVAILABILITY STATEMENT}

The raw data supporting the conclusions of this article will be made available by the authors, without undue reservation.

\section{ETHICS STATEMENT}

The studies involving human participants were reviewed and approved by the Carthage College Institutional Review

\section{REFERENCES}

Allen, M. T., Handy, J. D., Blankenship, M. R., and Servatius, R. J. (2018). The distressed (Type D) personality factor of social inhibition, but not negative affectivity, enhances eyeblink conditioning. Behav. Brain Res. 345, 93-103. doi: 10.1016/j.bbr.2018.02.035

Allen, M. T., Jameson, M. M., and Myers, C. E. (2017). Beyond behavioral inhibition: a computer avatar task designed to assess behavioral inhibition extends to harm avoidance. Front. Psychol. 8:1560. doi: 10.3389/fpsyg.2017. 01560

Allen, M. T., Myers, C. E., Beck, K. D., Pang, K. C. H., and Servatius, R. J. (2019). Inhibited personality temperaments translated through enhanced avoidance and associative learning increase vulnerability for PTSD. Front. Psychol. 10:496.

Allen, M. T., Myers, C. E., and Servatius, R. J. (2014). Avoidance prone individuals self reporting behavioral inhibition exhibit facilitated acquisition and altered extinction of conditioned eyeblinks with partial reinforcement schedules. Front. Behav. Neurosci. 8:347.

Allen, M. T., Myers, C. E., and Servatius, R. J. (2016). Uncertainty of trial timing enhances acquisition of conditioned eyeblinks in anxiety vulnerable individuals. Behav. Brain Res. 304, 86-91. doi: 10.1016/j.bbr.2016.02.007

Barlow, D. H. (2000). Unraveling the mysteries of anxiety and its disorders from the perspective of emotion theory. Am. Psychol. 55, 1247-1263. doi: 10.1037/ 0003-066x.55.11.1247

Beck, K. D., Jiao, X., Pang, K. C., and Servatius, R. J. (2010). Vulnerability factors in anxiety determined through differences in active-avoidance behavior. Prog. Neuropsychopharmacol. Biol. Psychiatry 34, 852-860. doi: 10.1016/j.pnpbp. 2010.03.036

Berntson, G. G., Bigger, J. T. Jr., Eckberg, D. L., Grossman, P., Kaufmann, P. G., Malik, M., et al. (1997). Heart rate variability: origins, methods, and interpretive caveats. Psychophysiology 34, 623-648. doi: 10.1111/j.1469-8986.1997.tb02140. $\mathrm{x}$

Bibbey, A., Carroll, D., Ginty, A. T., and Phillips, A. C. (2015). Cardiovascular and cortisol reactions to acute psychological stress under conditions of high versus low social evaluative threat: associations with the type D personality construct. Psychosom. Med. 77, 599-608. doi: 10.1097/psy.0000000000000194

Biederman, J., Hirshfeld-Becker, D. R., Rosenbaum, J. F., Herot, C., Friedman, D., Snidman, N., et al. (2001). Further evidence of association between behavioral inhibition and social anxiety in children. Am. J. Psychiatry 158, 1673-1679. doi: 10.1176/appi.ajp.158.10.1673
Board. The patients/participants provided their written informed consent to participate in this study.

\section{AUTHOR CONTRIBUTIONS}

RS, PM, and DM performed the design of the experiment. PM, DM, JM, and DC-S were responsible for IRB interactions and data collection. PM, DM, and JM accomplished the instrumentation. PM, DM, JM, and DC-S accomplished the data collation. RS, JH, and MA accomplished the data analyses. All authors contributed to manuscript preparation and editing.

\section{FUNDING}

This work was supported by the Stress and Motivated Behavior Institute through funding from the Department of Defense, United States Army Combat Capabilities Development Command (CCDC).

Brown, S. J., and Howden, R. (2008a). "The effects of a respiratory acidosis on human heart rate variability," in Integration in Respiratory Control: Advances in Experimental Medicine and Biology, Vol. 605, eds M. J. Poulin, and R. J. A. Wilson, (New York, NY: Springer), 361-365. doi: 10.1007/978-0-387-736938_63

Brown, S. J., Barnes, M. J., and Mundel, T. (2014). Effects of hypoxia and hypercapnia on human HRV and respiratory sinus arrhythmia. Acta Physiol. Hung. 101, 263-272. doi: 10.1556/aphysiol.101.2014.3.1

Brown, S. J., and Howden, R. (2008b). The effects of a respiratory acidosis on human heart rate variability. Adv. Exp. Med. Biol. 605, 361-365.

Brown, S. J., Mundel, T., and Brown, J. A. (2007). Cardiac vagal control and respiratory sinus arrhythmia during hypercapnia in humans. J. Physiol. Sci. 57, 337-342. doi: 10.2170/physiolsci.rp009407

Bystritsky, A., Craske, M., Maidenberg, E., Vapnik, T., and Shapiro, D. (2000). Autonomic reactivity of panic patients during a $\mathrm{CO} 2$ inhalation procedure. Depress Anxiety 11, 15-26. doi: 10.1002/(sici)1520-6394(2000)11:1<15::aidda3 > 3.0.co; $2-\mathrm{w}$

Caulfield, M. D., Vanmeenen, K. M., and Servatius, R. J. (2015). Facilitated acquisition of standard but not long delay classical eyeblink conditioning in behaviorally inhibited adolescents. Behav. Brain Res. 278, 476-481. doi: 10. 1016/j.bbr.2014.10.027

Chasiropoulou, C., Siouti, N., Mougiakos, T., and Dimitrakopoulos, S. (2019). The diathesis-stress model in the emergence of major psychiatric disorders during military service. Psychiatriki 30, 291-298. doi: 10.22365/jpsych.2019.304.291

Fox, N. A., and Pine, D. S. (2012). Temperament and the emergence of anxiety disorders. J. Am. Acad. Child Adoles. Psychiatry 51:125. doi: 10.1016/j.jaac.2011. 10.006

Friedman, B. H. (2007). An autonomic flexibility-neurovisceral integration model of anxiety and cardiac vagal tone. Biol. Psychol. 74, 185-199. doi: 10.1016/j. biopsycho.2005.08.009

Gladstone, G., and Parker, G. (2005). Measuring a behaviorally inhibited temperament style: development and initial validation of new self-report measures. Psychiatry Res. 135, 133-143. doi: 10.1016/j.psychres.2005. 03.005

Gladstone, G. L., Parker, G. B., Mitchell, P. B., Wilhelm, K. A., and Malhi, G. S. (2005). Relationship between self-reported childhood behavioral inhibition and lifetime anxiety disorders in a clinical sample. Depress Anxiety 22, 103-113. doi: 10.1002/da.20082 
Gorman, J. M., Fyer, M. R., Goetz, R., Askanazi, J., Liebowitz, M. R., Fyer, A. J., et al. (1988). Ventilatory physiology of patients with panic disorder. Arch. Gen. Psychiatry 45, 31-39. doi: 10.1001/archpsyc.1988.01800250035006

Guyenet, P. G., Abbott, S. B., and Stornetta, R. L. (2013). The respiratory chemoreception conundrum: light at the end of the tunnel? Brain Res. 1511, 126-137. doi: 10.1016/j.brainres.2012.10.028

Handy, J. D., Avcu, P., Ko, N., Ortiz, A., Doria, M. J., and Servatius, R. J. (2018). Facilitated acquisition of the classically conditioned eyeblink response in active duty military expressing posttraumatic stress disorder symptoms. Behav. Brain Res. 339, 106-113. doi: 10.1016/j.bbr.2017.11.014

Heathers, J. A. (2014). Everything Hertz: methodological issues in short-term frequency-domain HRV. Front. Physiol. 5:177.

Holloway, J. L., Trivedi, P., Myers, C. E., and Servatius, R. J. (2012). Enhanced conditioned eyeblink response acquisition and proactive interference in anxiety vulnerable individuals. Front. Behav. Neurosci. 6:76.

Huang, W.-L., Chang, L.-R., Kuo, T. B., Lin, Y.-H., Chen, Y.-Z., and Yang, C. C. (2013). Gender differences in personality and heart-rate variability. Psychiatry Res. 209, 652-657. doi: 10.1016/j.psychres.2013.01.031

Kaufmann, T., Sutterlin, S., Schulz, S. M., and Vogele, C. (2011). ARTiiFACT: a tool for heart rate artifact processing and heart rate variability analysis. Behav. Res. Methods 43, 1161-1170. doi: 10.3758/s13428-011-0107-7

Laborde, S., Mosley, E., and Thayer, J. F. (2017). Heart rate variability and cardiac vagal tone in psychophysiological research - recommendations for experiment planning, data analysis, and data reporting. Front. Psychol. 8:213.

Luque-Casado, A., Perales, J. C., Cardenas, D., and Sanabria, D. (2016). Heart rate variability and cognitive processing: the autonomic response to task demands. Biol. Psychol. 113, 83-90. doi: 10.1016/j.biopsycho.2015.11.013

Martin, L. A., Doster, J. A., Critelli, J. W., Lambert, P. L., Purdum, M., Powers, C., et al. (2010). Ethnicity and Type D personality as predictors of heart rate variability. Int. J. Psychophysiol. 76, 118-121. doi: 10.1016/j.ijpsycho.2010.03. 001

Mcauley, J., Stewart, A., Webber, E., Cromwell, H., Servatius, R., and Pang, K. (2009). Wistar-Kyoto rats as an animal model of anxiety vulnerability: support for a hypervigilance hypothesis. Behav. Brain Res. 204, 162-168. doi: 10.1016/j. bbr.2009.05.036

Mellick, W. H., Mills, J. A., Kroska, E. B., Calarge, C. A., Sharp, C., and Dindo, L. N. (2019). Experiential avoidance predicts persistence of major depressive disorder and generalized anxiety disorder in late adolescence. J. Clin. Psychiatry $80: 18 \mathrm{~m} 12265$.

Miller, J. R., Cook-Snyder, D., Buchholz, A., Evenhouse, A., Nicosia, T., Grove, A., et al. (2018). "Developing a low budget system to mix, store, and deliver enhanced respiratory gases for human research in liberal arts college setting," in Proceedings of the 1st Wisconsin Space Conference, Appleton, WI. Available online at: https://wsgc.carthage.edu/ojs/index.php/wsc/article/view/240

Myers, C. E., Vanmeenen, K. M., Mcauley, J. D., Beck, K. D., Pang, K. C., and Servatius, R. J. (2012a). Behaviorally inhibited temperament is associated with severity of post-traumatic stress disorder symptoms and faster eyeblink conditioning in veterans. Stress 15, 31-44. doi: 10.3109/10253890.2011.578184

Myers, C. E., Vanmeenen, K. M., and Servatius, R. J. (2012b). Behavioral inhibition and PTSD symptoms in veterans. Psychiatry Res. 196, 271-276. doi: 10.1016/j. psychres.2011.11.015

O’Donnell, M. L., Elliott, P., Lau, W., and Creamer, M. (2007). PTSD symptom trajectories: from early to chronic response. Behav. Res. Ther. 45, 601-606. doi: 10.1016/j.brat.2006.03.015

Pérez-Edgar, K., Bar-Haim, Y., Mcdermott, J. M., Chronis-Tuscano, A., Pine, D. S., and Fox, N. A. (2010). Attention biases to threat and behavioral inhibition in early childhood shape adolescent social withdrawal. Emotion 10:349. doi: $10.1037 / \mathrm{a} 0018486$

Perna, G., Romano, P., Caldirola, D., Cucchi, M., and Bellodi, L. (2003). Anxiety sensitivity and $35 \% \mathrm{CO} 2$ reactivity in patients with panic disorder. J. Psychosom. Res. 54, 573-577. doi: 10.1016/s0022-3999(02)00468-3

Puttonen, S., Elovainio, M., Kivimäki, M., Koskinen, T., Pulkki-Råback, L., Viikari, J. S., et al. (2008). Temperament, health-related behaviors, and autonomic cardiac regulation: the cardiovascular risk in young Finns study. Biol. Psychol. 78, 204-210. doi: 10.1016/j.biopsycho.2008.03.003

Quintana, D. S., Alvares, G. A., and Heathers, J. A. (2016). Guidelines for Reporting Articles on Psychiatry and Heart rate variability (GRAPH): recommendations to advance research communication. Transl. Psychiatry 6:e803. doi: 10.1038/tp. 2016.73

Quintana, D. S., and Heathers, J. A. (2014). Considerations in the assessment of heart rate variability in biobehavioral research. Front. Psychol. 5:805.

Ricart, T. M., De Niear, M. A., Jiao, X., Pang, K. C., Beck, K. D., and Servatius, R. J. (2011). Deficient proactive interference of eyeblink conditioning in Wistar-Kyoto rats. Behav. Brain Res. 216, 59-65. doi: 10.1016/j.bbr.2010. 07.005

Servatius, R. J., Handy, J. D., Doria, M. J., Myers, C. E., Marx, C. E., Lipsky, R., et al. (2017). Stress-related mental health symptoms in coast guard: incidence, vulnerability, and neurocognitive performance. Front. Psychol. 8:1513.

Servatius, R. J., Jiao, X., Beck, K. D., Pang, K. C., and Minor, T. R. (2008). Rapid avoidance acquisition in Wistar-Kyoto rats. Behav. Brain Res. 192, 191-197. doi: 10.1016/j.bbr.2008.04.006

Sheynin, J., Beck, K. D., Pang, K. C., Servatius, R. J., Shikari, S., Ostovich, J., et al. (2014). Behaviourally inhibited temperament and female sex, two vulnerability factors for anxiety disorders, facilitate conditioned avoidance (also) in humans. Behav. Proc. 103, 228-235. doi: 10.1016/j.beproc.2014.01.003

Sheynin, J., Moustafa, A. A., Beck, K. D., Servatius, R. J., and Myers, C. E. (2015). Testing the role of reward and punishment sensitivity in avoidance behavior: a computational modeling approach. Behav. Brain Res. 283, 121-138. doi: 10.1016/j.bbr.2015.01.033

Tapp, W., Servatius, R., Hunt, J., and Powell, D. A. (1997). Vagal activity predicts eyeblink conditioning in human subjects. Neuroreport 8, 1203-1207. doi: 10. 1097/00001756-199703240-00029

Tzeng, Y. C., Larsen, P. D., and Galletly, D. C. (2007). Effects of hypercapnia and hypoxemia on respiratory sinus arrhythmia in conscious humans during spontaneous respiration. Am. J. Physiol. Heart Circulat. Physiol. 292, H2397H2407.

Valenca, A. M., Nardi, A. E., Nascimento, I., Zin, W. A., and Versiani, M. (2002). Respiratory panic disorder subtype and sensitivity to the carbon dioxide challenge test. Braz. J. Med. Biol. Res. 35, 783-788. doi: 10.1590/s0100879x2002000700004

Van Den Hout, M. A., Griez, E., Van Der Molen, G. M., and Lousberg, H. (1987). Pulmonary carbon dioxide and panic-arousing sensations after $35 \%$ carbon dioxide inhalation: hypercapnia/hyperoxia versus hypercapnia/normoxia. J. Behav. Ther. Exp. Psychiatry 18, 19-23. doi: 10.1016/0005-7916(87)90067-x

White, L. K., Degnan, K. A., Henderson, H. A., Pérez-Edgar, K., Walker, O. L., Shechner, T., et al. (2017). Developmental relations among behavioral inhibition, anxiety, and attention biases to threat and positive information. Child Dev. 88, 141-155. doi: $10.1111 /$ cdev.12696

Young, F. L., and Leicht, A. S. (2011). Short-term stability of resting heart rate variability: influence of position and gender. Appl. Physiol. Nutr. Metab. 36, 210-218. doi: 10.1139/h10-103

Disclaimer: The views expressed in this article reflect the results of research conducted by the authors and do not necessarily reflect the official policy or position of the Department of Veterans Affairs, Department of the Navy, Department of Defense, nor the United States Government. RS and JH are employees of the United States Government. This work was prepared as part of their official duties. Title 17 U.S.C. $\$ 105$ provides that "Copyright protection under this title is not available for any work of the United States Government." Title 17 U.S.C. $\$ 101$ defines a United States Government work as a work prepared by a military service member or employee of the United States Government as part of that person's official duties.

Conflict of Interest: The authors declare that the research was conducted in the absence of any commercial or financial relationships that could be construed as a potential conflict of interest.

Copyright (c) 2020 Martino, Miller, Miller, Allen, Cook-Snyder, Handy and Servatius. This is an open-access article distributed under the terms of the Creative Commons Attribution License (CC BY). The use, distribution or reproduction in other forums is permitted, provided the original author(s) and the copyright owner(s) are credited and that the original publication in this journal is cited, in accordance with accepted academic practice. No use, distribution or reproduction is permitted which does not comply with these terms. 